CRYSTALLOGRAPHIC COMMUNICATIONS

ISSN 2056-9890

Received 17 May 2016

Accepted 31 May 2016

Edited by M. Weil, Vienna University of Technology, Austria

Keywords: crystal structure; silver thiocyanate; ammonia; argentophilicity.

CCDC reference: 1482850

Supporting information: this article has supporting information at journals.iucr.org/e

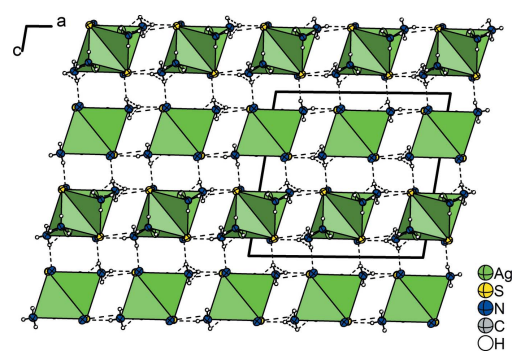

OPEN $\odot$ ACCESS

\section{Crystal structure of $\mathrm{Ag}_{2}(\mu-\mathrm{SCN})_{2}\left(\mathrm{NH}_{3}\right)_{4}$}

\author{
Thomas G. Müller and Florian Kraus*
}

Anorganische Chemie, Fluorchemie, Fachbereich Chemie, Philipps-Universität Marburg, Hans-Meerwein-Strasse 4, 35032 Marburg, Germany. *Correspondence e-mail: florian.kraus@chemie.uni-marburg.de

Di- $\mu$-thiocyanato-bis[diamminesilver(I)], $\left[\mathrm{Ag}_{2}(\mu \text {-SCN })_{2}\left(\mathrm{NH}_{3}\right)_{4}\right]$, was synthesized by the reaction of $\mathrm{AgSCN}$ with anhydrous liquid ammonia. In the binuclear molecule, the $\mathrm{Ag}^{\mathrm{I}}$ atom is coordinated by two ammine ligands and the $\mathrm{S}$ atom of one thiocyanate ligand. Two of these $\left[\mathrm{Ag}(\mathrm{SCN})\left(\mathrm{NH}_{3}\right)_{2}\right]$ units are bridged by the $\mathrm{S}$ atoms of the thiocyanate anions at longer distances, leading to a dimer with point group symmetry $C_{2}$. The distance between the $\mathrm{Ag}^{\mathrm{I}}$ atoms in the dimer is at 3.0927 (6) $\AA$ within the range of argentophilic interactions. The crystal structure displays $\mathrm{N}-\mathrm{H} \cdots \mathrm{N}$ and $\mathrm{N}-\mathrm{H} \cdots \mathrm{S}$ hydrogen-bonding interactions that build up a three-dimensional network.

\section{Chemical context}

The reactions of various silver salts with liquid ammonia and their products are in almost all cases still unknown. In textbooks, the formation of the linear diamminesilver(I) cation is often predicted without any structural evidence. In this contribution we want to report on the reaction and the product of $\mathrm{AgSCN}$ with liquid ammonia at $237 \mathrm{~K}$. A dinuclear $\mathrm{Ag}^{\mathrm{I}}$ complex was obtained.

$2 \mathrm{AgSCN}+4 \mathrm{NH}_{3} \underset{\mathrm{NH}_{3(l)}}{\stackrel{237 \mathrm{~K}}{\longrightarrow}} \mathrm{Ag}_{2}(\mu-\mathrm{SCN})_{2}\left(\mathrm{NH}_{3}\right)_{4}$

\section{Structural commentary}

All atoms are located on general sites. The silver atom Ag1 is surrounded by two ammine ligands (N2 and N3) with distances of 2.269 (2) and 2.248 (2) $\AA$, respectively. These values are in good agreement with other reported $\mathrm{Ag}-\mathrm{N}$ distances (Zachwieja \& Jacobs, 1989). The thiocyanate anion coordinates with its soft sulfur atom to the silver atom at a distance of 2.5363 (6) $\AA$, which is similar compared to those of pure AgSCN (Lindqvist, 1957). The $\mathrm{S}-\mathrm{C}-\mathrm{N}$ angle in this pseudo-halide anion is with $178.2(2)^{\circ}$ almost linear. Two of the $\left[\mathrm{Ag}(\mathrm{SCN})\left(\mathrm{NH}_{3}\right)_{2}\right]$ units are connected to each other via bridging $\mathrm{S}$ atoms of the thiocyanato ligands into a dimer located about a twofold rotation axis (Fig. 1). The resulting coordination polyhedron around $\mathrm{Ag} 1$ is that of a distorted tetrahedron where one short Ag-S distance [2.5363 (6) $\AA$ ] and a long one [3.0533 (7) $\AA$ ] are observed. Therefore, the bond towards the latter may be regarded as weaker. In the dimer, the two tetrahedra are connected through one edge into a double tetrahedron. It is interesting to note that the two $\mathrm{SCN}^{-}$anions point in the same direction as there is no center of inversion within the molecule but only the twofold rotation axis of the space-group type. The Ag. $\cdots A g$ distance is short at 


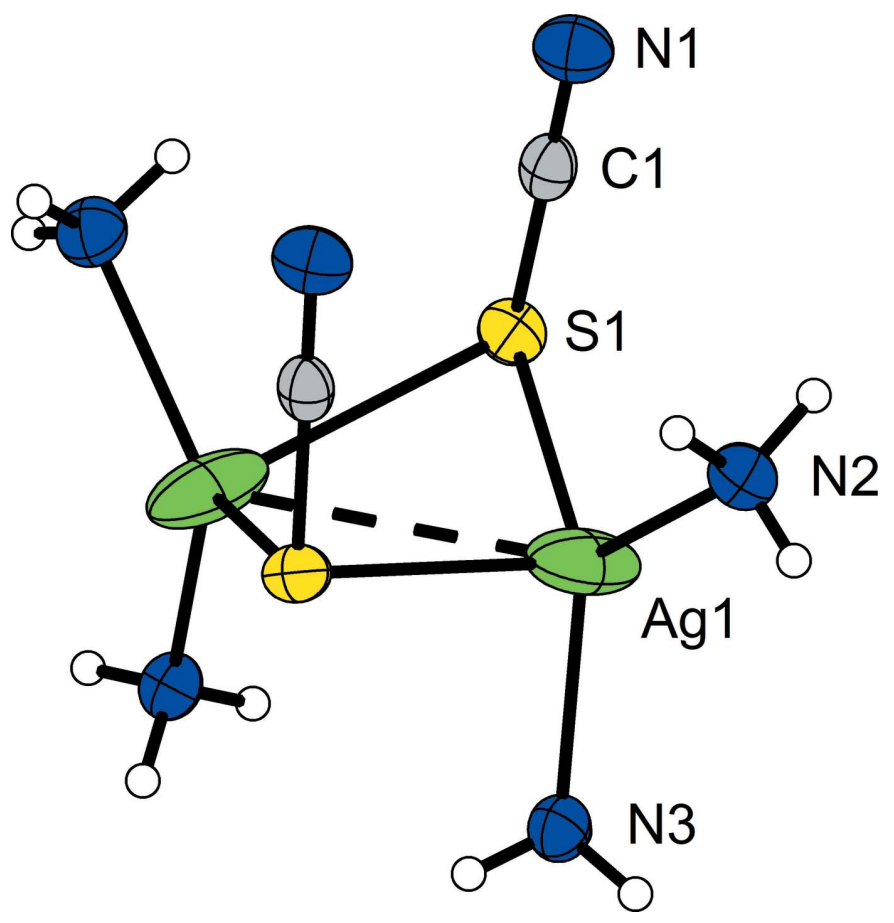

Figure 1

The dimeric $\left[\mathrm{Ag}(\mathrm{SCN})\left(\mathrm{NH}_{3}\right)_{2}\right]_{2}$ unit in the title compound. Displacement ellipsoids are shown at the $70 \%$ probability level and $\mathrm{H}$ atoms are drawn with an arbitrary radius. All non-labelled atoms are generated by symmetry code $\left(-x, y,-z+\frac{1}{2}\right)$.

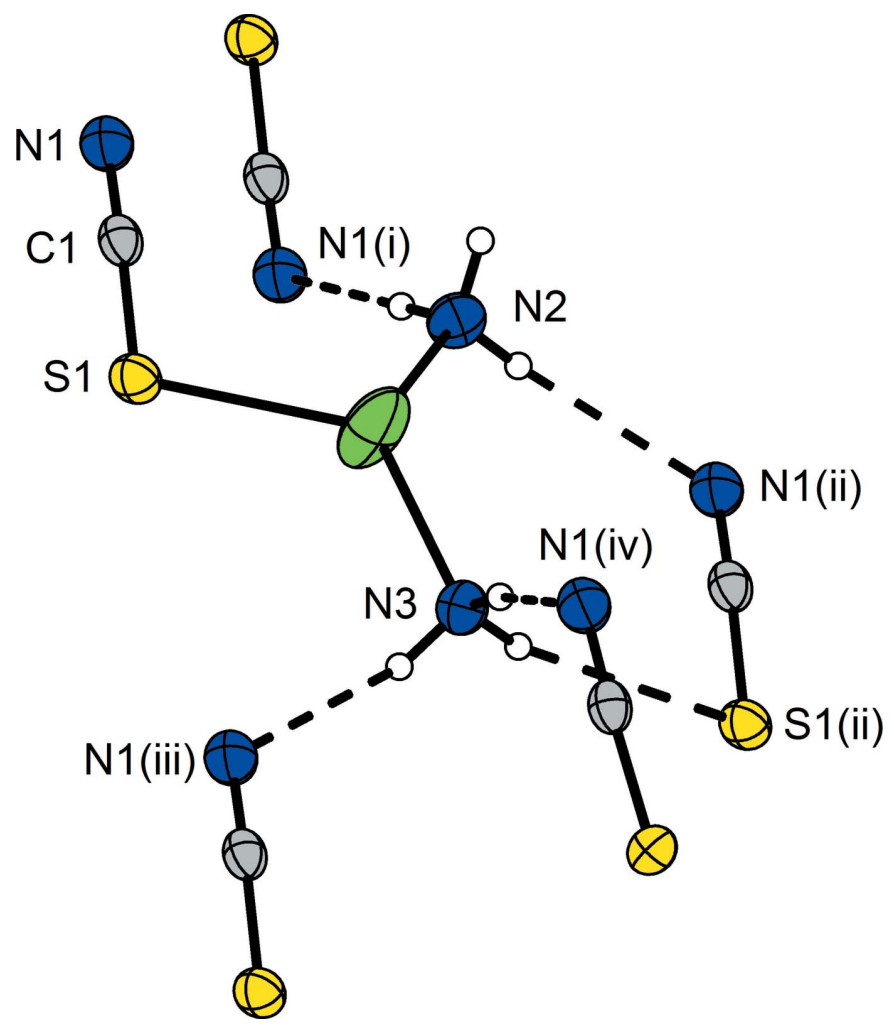

Figure 2

The hydrogen bonds (dashed lines) present in the structure of the title compound as illustrated for one $\left[\mathrm{Ag}(\mathrm{SCN})\left(\mathrm{NH}_{3}\right)_{2}\right]$ unit with the acceptor groups of four surrounding molecules. Displacement ellipsoids as in Fig. 1. [Symmetry codes: (i) $-x,-y+1,-z$; (ii) $x+\frac{1}{2}, y-\frac{1}{2}, z$; (iii) $x, y-1, z$; (iv) $-x, y-1,-z+\frac{1}{2}$.]
Table 1

Hydrogen-bond geometry $\left(\AA,^{\circ}\right)$.

\begin{tabular}{lllll}
\hline$D-\mathrm{H} \cdots A$ & $D-\mathrm{H}$ & $\mathrm{H} \cdots A$ & $D \cdots A$ & $D-\mathrm{H} \cdots A$ \\
\hline $\mathrm{N} 2-\mathrm{H} 2 C \cdots \mathrm{N} 1^{\mathrm{i}}$ & $0.89(4)$ & $2.34(4)$ & $3.230(3)$ & $171(3)$ \\
$\mathrm{N} 2-\mathrm{H} 2 B \cdots \mathrm{N} 1^{1 i}$ & $0.83(5)$ & $2.43(5)$ & $3.255(3)$ & $170(4)$ \\
$\mathrm{N} 3-\mathrm{H} 3 C \cdots \mathrm{N} 1^{\mathrm{iii}}$ & $0.90(4)$ & $2.31(4)$ & $3.128(3)$ & $151(3)$ \\
$\mathrm{N} 3-\mathrm{H} 3 B \cdots \mathrm{N} 1^{\text {iv }}$ & $0.83(4)$ & $2.39(4)$ & $3.208(3)$ & $168(4)$ \\
$\mathrm{N} 3-\mathrm{H} 3 A \cdots \mathrm{S} 1^{\mathrm{ii}}$ & $0.87(4)$ & $2.82(4)$ & $3.672(2)$ & $166(3)$ \\
\hline Symmetry codes: (i) & $-x,-y+1,-z ;$ & (ii) $x+\frac{1}{2}, y-\frac{1}{2}, z ;$ (iii) & $x, y-1, z ;$ (iv) \\
$-x, y-1,-z+\frac{1}{2}$. & & &
\end{tabular}

3.0927 (6) $\AA$, and is clearly in the range of argentophilic interactions (Jansen, 1987; Zachwieja \& Jacobs, 1989; Schmidbaur \& Schier, 2015).

\section{Supramolecular features}

The dinuclear complexes are connected to others via hydrogen bonds between the ammine ligands ( $\mathrm{N} 2$ and $\mathrm{N} 3$ ) as donors and the $\mathrm{N} 1$ and $\mathrm{S} 1$ atoms of the thiocyanato ligand as acceptors. A three-dimensional network is formed in which each $\left[\mathrm{Ag}(\mathrm{SCN})\left(\mathrm{NH}_{3}\right)_{2}\right]$ unit is coordinated by four (Fig. 2) and the dimer by eight other molecules. Six are arranged like a hexagon around the central molecule with all SCN ligands pointing in the same direction. Two molecules reside above and below this fictitious plane and are shifted towards a corner of the hexagon whereby the SCN ligands point in the opposite direction. Each of these two molecules shows the same coordination as described above, and overall, an $A B$-stacking of the molecules along [001], similar to the hexagonal closest packing, is obtained. The crystal structure is shown in Fig. 3. It should be noted that no acceptor atom for the hydrogen atom $\mathrm{H} 2 \mathrm{~A}$ is present in the neighbourhood within the range of the sum of the van der Waals radii of $\mathrm{H}$ and $\mathrm{N}$ atoms. Numerical details of the hydrogen bonding are given in Table 1.

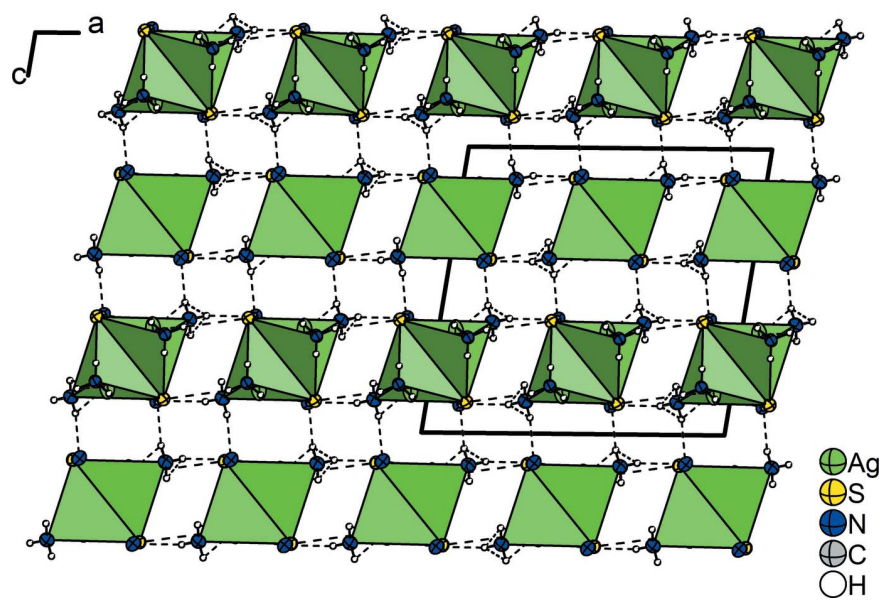

Figure 3

The crystal structure of $\mathrm{Ag}_{2}(\mathrm{SCN})_{2}\left(\mathrm{NH}_{3}\right)_{4}$ viewed along [010] with hydrogen bonds (dashed lines). Displacement ellipsoids as in Fig.1. 
Table 2

Experimental details.

\begin{tabular}{|c|c|}
\hline \multicolumn{2}{|l|}{ Crystal data } \\
\hline Chemical formula & {$\left[\mathrm{Ag}_{2}(\mathrm{SCN})_{2}\left(\mathrm{NH}_{3}\right)_{4}\right]$} \\
\hline$M_{\mathrm{r}}$ & 400.04 \\
\hline Crystal system, space group & Monoclinic, $C 2 / c$ \\
\hline Temperature (K) & 100 \\
\hline$a, b, c(\AA)$ & $12.8263(9), 7.1879(3), 12.2478(9)$ \\
\hline$\beta\left({ }^{\circ}\right)$ & $98.936(6)$ \\
\hline$V\left(\AA^{6}\right)$ & $1115.47(12)$ \\
\hline$Z$ & 4 \\
\hline Radiation type & Mo $K \alpha$ \\
\hline$\mu\left(\mathrm{mm}^{-1}\right)$ & 3.85 \\
\hline Crystal size (mm) & $0.26 \times 0.16 \times 0.14$ \\
\hline \multicolumn{2}{|l|}{ Data collection } \\
\hline Diffractometer & Stoe IPDS 2T \\
\hline Absorption correction & $\begin{array}{l}\text { Numerical }(X-R E D 32 \text { and } \\
\quad X \text {-SHAPE; Stoe \& Cie, 2009) }\end{array}$ \\
\hline$T_{\min }, T_{\max }$ & $0.768,0.918$ \\
\hline $\begin{array}{l}\text { No. of measured, independent and } \\
\text { observed }[I>2 \sigma(I)] \text { reflections }\end{array}$ & $7257,1690,1593$ \\
\hline$R_{\text {int }}$ & 0.028 \\
\hline$(\sin \theta / \lambda)_{\max }\left(\AA^{-1}\right)$ & 0.714 \\
\hline \multicolumn{2}{|l|}{ Refinement } \\
\hline$R\left[F^{2}>2 \sigma\left(F^{2}\right)\right], w R\left(F^{2}\right), S$ & $0.029,0.061,1.11$ \\
\hline No. of reflections & 1690 \\
\hline No. of parameters & 79 \\
\hline H-atom treatment & All H-atom parameters refined \\
\hline$\Delta \rho_{\max }, \Delta \rho_{\min }\left(\mathrm{e} \AA^{-3}\right)$ & $1.26,-1.82$ \\
\hline
\end{tabular}

Computer programs: $X$-AREA (Stoe \& Cie, 20011), X-RED32 (Stoe \& Cie, 2009), SHELXT (Sheldrick, 2015a), SHELXL2014 (Sheldrick, 2015b), SHELXLE (Hübschle et al., 2011), DIAMOND (Brandenburg, 2015) and publCIF (Westrip, 2010).

\section{Synthesis and crystallization}

$400 \mathrm{mg}$ (2.41 mmol) of AgSCN were placed in a flame-dried Schlenk tube under argon. Approximately $0.5 \mathrm{ml}$ of liquid ammonia were condensed into the reaction vessel. The reaction vessel was stored at $237 \mathrm{~K}$. After two weeks, colorless crystals of suitable size for X-ray diffraction were obtained from the colorless solution. The formation of the title compound is shown in the scheme.

\section{Refinement}

Crystal data, data collection and structure refinement details are summarized in Table 2. All hydrogen atoms of the ammine ligands were located from a difference Fourier map and were refined isotropically without further restraints.

\section{Acknowledgements}

The authors would like to thank Hendrik Borkowski for his preparative work. FK thanks the Deutsche Forschungsgemeinschaft for his Heisenberg professorship.

\section{References}

Brandenburg, K. (2015). DIAMOND. Crystal Impact GbR, Bonn, Germany.

Hübschle, C. B., Sheldrick, G. M. \& Dittrich, B. (2011). J. Appl. Cryst. 44, 1281-1284.

Jansen, M. (1987). Angew. Chem. 99, 1136-1149.

Lindqvist, I. (1957). Acta Cryst. 10, 29-32.

Schmidbaur, H. \& Schier, A. (2015). Angew. Chem. 127, 756-797.

Sheldrick, G. M. (2015a). Acta Cryst. A71, 3-8.

Sheldrick, G. M. (2015b). Acta Cryst. C71, 3-8.

Stoe \& Cie (2009). X-RED32 and X-SHAPE. Stoe \& Cie GmbH, Darmstadt, Germany.

Stoe \& Cie (2011). X-AREA. Stoe \& Cie GmbH, Darmstadt, Germany.

Westrip, S. P. (2010). J. Appl. Cryst. 43, 920-925.

Zachwieja, U. \& Jacobs, H. (1989). Z. Anorg. Allg. Chem. 571, 37-50. 


\section{supporting information}

Acta Cryst. (2016). E72, 881-883 [https://doi.org/10.1107/S2056989016008823]

\section{Crystal structure of $\mathrm{Ag}_{2}(\mu-\mathrm{SCN})_{2}\left(\mathrm{NH}_{3}\right)_{4}$}

\section{Thomas G. Müller and Florian Kraus}

\section{Computing details}

Data collection: $X$-AREA (Stoe \& Cie, 2011); cell refinement: $X$-AREA (Stoe \& Cie, 2011); data reduction: $X$-RED32 (Stoe \& Cie, 2009); program(s) used to solve structure: SHELXT (Sheldrick, 2015a); program(s) used to refine structure: SHELXL2014 (Sheldrick, 2015b) and SHELXLE (Hübschle et al., 2011); molecular graphics: DIAMOND (Brandenburg, 2015); software used to prepare material for publication: publCIF (Westrip, 2010).

Di- $\mu$-thiocyanato-bis[diamminesilver(I)]

Crystal data

$\left[\mathrm{Ag}_{2}(\mathrm{SCN})_{2}\left(\mathrm{NH}_{3}\right)_{4}\right]$

$M_{r}=400.04$

Monoclinic, $C 2 / c$

$a=12.8263(9) \AA$

$b=7.1879(3) \AA$

$c=12.2478(9) \AA$

$\beta=98.936(6)^{\circ}$

$V=1115.47(12) \AA^{3}$

$Z=4$

Data collection

Stoe IPDS 2T diffractometer

Radiation source: sealed X-ray tube, 12 x 0.4 $\mathrm{mm}$ long-fine focus

Plane graphite monochromator

Detector resolution: 6.67 pixels $\mathrm{mm}^{-1}$

rotation method scans

Absorption correction: numerical

(X-RED32 and X-SHAPE; Stoe \& Cie, 2009)

\section{Refinement}

Refinement on $F^{2}$

Least-squares matrix: full

$R\left[F^{2}>2 \sigma\left(F^{2}\right)\right]=0.029$

$w R\left(F^{2}\right)=0.061$

$S=1.11$

1690 reflections

79 parameters

0 restraints
$F(000)=768$

$D_{\mathrm{x}}=2.382 \mathrm{Mg} \mathrm{m}^{-3}$

Mo $K \alpha$ radiation, $\lambda=0.71073 \AA$

Cell parameters from 13119 reflections

$\theta=3.2-35.2^{\circ}$

$\mu=3.85 \mathrm{~mm}^{-1}$

$T=100 \mathrm{~K}$

Block, colorless

$0.26 \times 0.16 \times 0.14 \mathrm{~mm}$

$T_{\min }=0.768, T_{\max }=0.918$

7257 measured reflections

1690 independent reflections

1593 reflections with $I>2 \sigma(I)$

$R_{\text {int }}=0.028$

$\theta_{\text {max }}=30.5^{\circ}, \theta_{\min }=3.2^{\circ}$

$h=-18 \rightarrow 18$

$k=-10 \rightarrow 9$

$l=-17 \rightarrow 17$

Hydrogen site location: difference Fourier map

All $\mathrm{H}$-atom parameters refined

$w=1 /\left[\sigma^{2}\left(F_{\mathrm{o}}^{2}\right)+(0.0131 P)^{2}+6.0057 P\right]$

where $P=\left(F_{\mathrm{o}}{ }^{2}+2 F_{\mathrm{c}}{ }^{2}\right) / 3$

$(\Delta / \sigma)_{\max }<0.001$

$\Delta \rho_{\max }=1.26 \mathrm{e} \AA^{-3}$

$\Delta \rho_{\min }=-1.82$ e $\AA^{-3}$ 


\section{Special details}

Geometry. All esds (except the esd in the dihedral angle between two 1.s. planes) are estimated using the full covariance matrix. The cell esds are taken into account individually in the estimation of esds in distances, angles and torsion angles; correlations between esds in cell parameters are only used when they are defined by crystal symmetry. An approximate (isotropic) treatment of cell esds is used for estimating esds involving l.s. planes.

Fractional atomic coordinates and isotropic or equivalent isotropic displacement parameters $\left(\AA^{2}\right)$

\begin{tabular}{lllll}
\hline & $x$ & $y$ & $z$ & $U_{\text {iso }} * / U_{\text {eq }}$ \\
\hline Ag1 & $0.06012(2)$ & $0.01303(3)$ & $0.14990(3)$ & $0.04102(10)$ \\
S1 & $-0.13234(4)$ & $0.10809(8)$ & $0.10715(5)$ & $0.01844(11)$ \\
N1 & $-0.11243(17)$ & $0.4982(3)$ & $0.10139(18)$ & $0.0226(4)$ \\
N2 & $0.17384(18)$ & $0.2371(3)$ & $0.11205(19)$ & $0.0217(4)$ \\
N3 & $0.10144(16)$ & $-0.2907(3)$ & $0.16630(18)$ & $0.0189(4)$ \\
C1 & $-0.11906(16)$ & $0.3371(3)$ & $0.10457(17)$ & $0.0162(4)$ \\
H2A & $0.187(3)$ & $0.315(6)$ & $0.169(3)$ & $0.037(10)^{*}$ \\
H2B & $0.228(3)$ & $0.181(7)$ & $0.101(4)$ & $0.050(12)^{*}$ \\
H2C & $0.150(3)$ & $0.311(5)$ & $0.055(3)$ & $0.034(9)^{*}$ \\
H3A & $0.158(3)$ & $-0.322(5)$ & $0.140(3)$ & $0.029(9)^{*}$ \\
H3B & $0.110(3)$ & $-0.331(5)$ & $0.231(3)$ & $0.033(9)^{*}$ \\
H3C & $0.050(3)$ & $-0.355(6)$ & $0.125(3)$ & $0.039(10)^{*}$ \\
\hline
\end{tabular}

Atomic displacement parameters $\left(\AA^{2}\right)$

\begin{tabular}{lllllll}
\hline & $U^{11}$ & $U^{22}$ & $U^{33}$ & $U^{12}$ & $U^{13}$ & $U^{23}$ \\
\hline Ag1 & $0.02898(12)$ & $0.02005(11)$ & $0.0765(2)$ & $0.00256(8)$ & $0.01589(11)$ & $0.01630(11)$ \\
S1 & $0.0185(2)$ & $0.0165(2)$ & $0.0201(2)$ & $-0.00265(19)$ & $0.00216(18)$ & $0.00010(19)$ \\
N1 & $0.0216(9)$ & $0.0201(10)$ & $0.0247(10)$ & $0.0003(7)$ & $-0.0008(7)$ & $-0.0002(8)$ \\
N2 & $0.0245(10)$ & $0.0190(9)$ & $0.0220(10)$ & $0.0006(8)$ & $0.0048(8)$ & $0.0022(8)$ \\
N3 & $0.0177(9)$ & $0.0204(9)$ & $0.0191(9)$ & $0.0000(7)$ & $0.0045(7)$ & $0.0008(7)$ \\
C1 & $0.0139(9)$ & $0.0217(10)$ & $0.0126(8)$ & $0.0000(7)$ & $0.0009(7)$ & $-0.0011(8)$ \\
\hline
\end{tabular}

Geometric parameters $\left(\AA,^{\circ}\right)$

\begin{tabular}{llll}
\hline $\mathrm{Ag} 1-\mathrm{N} 3$ & $2.248(2)$ & $\mathrm{N} 2-\mathrm{H} 2 \mathrm{~A}$ & $0.89(4)$ \\
$\mathrm{Ag} 1-\mathrm{N} 2$ & $2.269(2)$ & $\mathrm{N} 2-\mathrm{H} 2 \mathrm{~B}$ & $0.83(5)$ \\
$\mathrm{Ag} 1-\mathrm{S} 1$ & $2.5363(6)$ & $\mathrm{N} 2-\mathrm{H} 2 \mathrm{C}$ & $0.89(4)$ \\
$\mathrm{Ag} 1-\mathrm{S} 1^{\mathrm{i}}$ & $3.0533(7)$ & $\mathrm{N} 3-\mathrm{H} 3 \mathrm{~A}$ & $0.87(4)$ \\
$\mathrm{Ag} 1-\mathrm{Ag} 1^{\mathrm{i}}$ & $3.0927(6)$ & $\mathrm{N} 3-\mathrm{H} 3 \mathrm{~B}$ & $0.83(4)$ \\
$\mathrm{S} 1-\mathrm{C} 1$ & $1.656(2)$ & $\mathrm{N} 3-\mathrm{H} 3 \mathrm{C}$ & $0.90(4)$ \\
$\mathrm{N} 1-\mathrm{C} 1$ & $1.162(3)$ & & \\
& & & $115(2)$ \\
$\mathrm{N} 3-\mathrm{Ag} 1-\mathrm{N} 2$ & $123.89(8)$ & $\mathrm{Ag} 1-\mathrm{N} 2-\mathrm{H} 2 \mathrm{C}$ & $104(3)$ \\
$\mathrm{N} 3-\mathrm{Ag} 1-\mathrm{S} 1$ & $119.24(6)$ & $\mathrm{H} 2 \mathrm{~A}-\mathrm{N} 2-\mathrm{H} 2 \mathrm{C}$ & $111(4)$ \\
$\mathrm{N} 2-\mathrm{Ag} 1-\mathrm{S} 1$ & $113.74(6)$ & $\mathrm{H} 2 \mathrm{~B}-\mathrm{N} 2-\mathrm{H} 2 \mathrm{C}$ & $115(2)$ \\
$\mathrm{N} 3-\mathrm{Ag} 1-\mathrm{Ag} 1^{\mathrm{i}}$ & $93.95(5)$ & $\mathrm{Ag} 1-\mathrm{N} 3-\mathrm{H} 3 \mathrm{~A}$ & $115(3)$ \\
$\mathrm{N} 2-\mathrm{Ag} 1-\mathrm{Ag} 1^{\mathrm{i}}$ & $125.28(6)$ & $\mathrm{Ag} 1-\mathrm{N} 3-\mathrm{H} 3 \mathrm{~B}$ & $105(3)$ \\
$\mathrm{S} 1-\mathrm{Ag} 1-\mathrm{Ag} 1^{\mathrm{i}}$ & $64.820(16)$ & $\mathrm{H} 3 \mathrm{~A}-\mathrm{N} 3-\mathrm{H} 3 \mathrm{~B}$ &
\end{tabular}




$\begin{array}{llll}\mathrm{C} 1-\mathrm{S} 1-\mathrm{Ag} 1 & 99.91(8) & \mathrm{Ag} 1-\mathrm{N} 3-\mathrm{H} 3 \mathrm{C} & 108(3) \\ \mathrm{Ag} 1-\mathrm{N} 2-\mathrm{H} 2 \mathrm{~A} & 109(3) & \mathrm{H} 3 \mathrm{~A}-\mathrm{N} 3-\mathrm{H} 3 \mathrm{C} & 104(3) \\ \mathrm{Ag} 1-\mathrm{N} 2-\mathrm{H} 2 \mathrm{~B} & 105(3) & \mathrm{H} 3 \mathrm{~B}-\mathrm{N} 3-\mathrm{H} 3 \mathrm{C} & 109(3) \\ \mathrm{H} 2 \mathrm{~A}-\mathrm{N} 2-\mathrm{H} 2 \mathrm{~B} & 113(4) & \mathrm{N} 1-\mathrm{C} 1-\mathrm{S} 1 & 178.2(2)\end{array}$

Symmetry code: (i) $-x, y,-z+1 / 2$.

Hydrogen-bond geometry $\left(\AA,{ }^{\circ}\right)$

\begin{tabular}{lllll}
\hline$D-\mathrm{H} \cdots A$ & $D-\mathrm{H}$ & $\mathrm{H} \cdots A$ & $D \cdots A$ & $D-\mathrm{H} \cdots A$ \\
\hline $\mathrm{N} 2-\mathrm{H} 2 C \cdots \mathrm{N} 1^{\text {ii }}$ & $0.89(4)$ & $2.34(4)$ & $3.230(3)$ & $171(3)$ \\
$\mathrm{N} 2-\mathrm{H} 2 B \cdots \mathrm{N} 1^{\mathrm{iii}}$ & $0.83(5)$ & $2.43(5)$ & $3.255(3)$ & $170(4)$ \\
$\mathrm{N} 3-\mathrm{H} 3 C \cdots \mathrm{N} 1^{\text {iv }}$ & $0.90(4)$ & $2.31(4)$ & $3.128(3)$ & $151(3)$ \\
$\mathrm{N} 3-\mathrm{H} 3 B \cdots \mathrm{N} 1^{\mathrm{v}}$ & $0.83(4)$ & $2.39(4)$ & $3.208(3)$ & $168(4)$ \\
$\mathrm{N} 3-\mathrm{H} 3 A \cdots \mathrm{S} 1^{\mathrm{iii}}$ & $0.87(4)$ & $2.82(4)$ & $3.672(2)$ & $166(3)$
\end{tabular}

Symmetry codes: (ii) $-x,-y+1,-z$; (iii) $x+1 / 2, y-1 / 2, z$; (iv) $x, y-1, z$; (v) $-x, y-1,-z+1 / 2$. 